\title{
Calibration of multilayer mirrors for the Multi-Spectral Solar Telescope Array II
}

\author{
Charles C. Kankelborg, James E. Plummer, Dennis S. Martínez-Galarce, \\ Ray H. O'Neal, Craig E. DeForest, Arthur B.C. Walker, Jr. \\ Center for Space Science and Astrophysics and Departments of Physics, \\ Applied Physics and Electrical Engineering \\ Stanford University \\ Stanford, California 94305 \\ Troy W. Barbee, Jr., J. W. Weed \\ Lawrence Livermore National Laboratory \\ Livermore, California 94550 \\ Richard B. Hoover \\ Space Science Laboratory, NASA Marshall Space Flight Center \\ Huntsville, Alabama 35812 \\ Forbes R. Powell \\ Luxel Corporation \\ Friday Harbor, Washington 98250
}

\begin{abstract}
The Multi-Spectral Solar Telescope Array II (MSSTA II), a rocket-borne solar observatory, was successfully flown on November 3, 1994 obtaining solar images in multiple XUV and FUV bands with an array of compact multilayer telescopes. Extensive measurements have recently been carried out on some of the multilayer telescopes at the Stanford Synchrotron Radiation Laboratory. These measurements are the first high spectral resolution calibrations of newly introduced MSSTA II instruments and instruments with $\lambda_{0}<130 \AA$. Previous measurements and/or calculations of telescope throughputs have been confirmed with greater accuracy. Results are presented on $\mathrm{Mo} / \mathrm{Si}$ multilayer bandpasses, and multilayer bandpass changes with time.
\end{abstract}

\section{INTRODUCTION}

We report here on the performance of multilayer mirrors developed for the Multi-Spectral Solar Telescope Array II (MSSTA II). The MSSTA II is a comprehensive rocket-borne solar observatory capable of obtaining narrow-band images of chromospheric, transition region, and coronal structures with very high angular resolution. High angular resolution imaging via normal incidence reflection is obtained by the use of multilayer mirrors. Multilayer optics have been successfully fabricated to permit the efficient reflection of radiation at normal incidence in the interval $40 \AA<\lambda<400 \AA$. In previous reviews, we have discussed the optical configurations that can be used for astronomical observations with multilayer optics ${ }^{1}$, reviewed the results of observations of the sun with multilayer optics $^{2}$; and discussed the spectral resolving power of multilayer optics ${ }^{3}$.

Calibration of the MSSTA II instruments is critical to the analysis of the flight data. The extraction of absolute flux measurements from the images necessitates accurate data on the absolute multilayer reflectivities, the throughputs of the XUV thin film filters used, and the response of the photographic films. We present the calibration of the multilayer reflectivities the present paper; calibration of the thin film filters is described by Plummer $e t a l^{4}$; calibration of the films used to record the MSSTA images is described by Hoover et al ${ }^{5}$. These measurements will 
enable us to calculate temperatures and electron densities of the many features of interest in the images obtained. An important characteristic of the MSSTA II payload is its ability to provide spatially resolved temperature information by its imaging of specific spectral lines. The temperature response of the telescopes is quite sensitive to telescope bandpass because of the high density of lines in the coronal spectrum. Calculations of temperature responses for the various systems based on previous measurements of telescopes and theoretical filter data are reported in DeForest $e t$ $a l^{6}$. High resolution measurements of telescope bandpasses throughout the full spectral range of MSSTA II insturmentation will enable us to improve our temperature response calculations, improving the capabilities of the MSSTA II as a powerful temperature diagnostic tool.

\section{REVIEW OF PREVIOUS MEASUREMENTS}

During March, April and May of 1992, most of the optical elements of the MSSTA payload were calibrated at the Stanford Synchrotron Radiation Laboratory (SSRL) on beam line 1-2. During March to May of 1993, further work was performed both in redoing difficult measurements with an improved setup and also in studying newly fabricated mirrors and filters. The beamline 1-2 monochromator has a high energy limit of approximately $250 \mathrm{eV}$ ( $\sim 50 \AA)$, making it impossible to make measurements in the bandpass of the $44.1 \AA$ Herschelian pair. Summaries of our previous results are shown in table 1. For the double-reflection Ritchey-Chrétien and Cassegrain doublereflection telescopes, the numbers given are for both mirrors as a system.

Table 1. Previous measurements of MSSTA II mirrors.

\begin{tabular}{|c|c|c|c|c|c|c|}
\hline Telescope & $\begin{array}{l}\text { Mirror coating } \\
\text { materials }\end{array}$ & 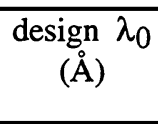 & $\begin{array}{c}\text { Measured } \\
\lambda_{0}(\AA)\end{array}$ & 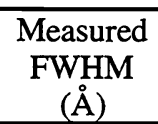 & $\begin{array}{c}\text { Measured } \\
\mathrm{R}_{0}(\%)\end{array}$ & Comments \\
\hline Ritchey-Chrétien II & $\mathrm{Mo} / \mathrm{Si}$ & 193 & 192.1 & 10.8 & 7.3 & $\mathrm{C}$ \\
\hline Ritchey-Chrétien III & $\mathrm{Mo} / \mathrm{Si}$ & 150 & 152.5 & 6.0 & 19.1 & $\mathrm{C}, \mathrm{N}$ \\
\hline Ritchey-Chrétien V & $\mathrm{Mo} / \mathrm{Mg}_{2} \mathrm{Si}$ & 304 & 309 & 22.3 & 4.1 & $\mathrm{C}$ \\
\hline Ritchey-Chrétien VII & $\mathrm{Al} / \mathrm{Mg}_{2} \mathrm{~F} / \mathrm{Os}$ & 1550 & - & 200 & 62 & $\mathrm{D}$ \\
\hline Cassegrain 1 & $\mathrm{Mo} / \mathrm{Si}$ & 173 & 173.5 & 7.5 & 11.8 & $A, B$ \\
\hline Cassegrain 2 & $\mathrm{Mo} / \mathrm{Si}$ & 211 & 210 & 17 & 3.5 & B \\
\hline Large Herschelian A & $\mathrm{Mo} / \mathrm{Si}$ & 193 & $183 / 187 / 193$ & $21 / 13.5 / 15$ & $33 / 41 / 34$ & $\mathrm{~A}, \mathrm{~B}, \mathrm{Z}$ \\
\hline Small Herschelian $\alpha$ & $\mathrm{Mo} / \mathrm{Si}$ & 143.3 & 146.8 & 5.1 & 28.6 & $\mathrm{C}$ \\
\hline Small Herschelian $\beta$ & WC/C & 54.7 & - & - & - & $A, N$ \\
\hline Small Herschelian $\delta$ & $\mathrm{Mo} / \mathrm{Si}$ & 150 & - & - & 一 & $\mathrm{A}, \mathrm{N}$ \\
\hline Small Herschelian $\varepsilon$ & $\mathrm{Mo} / \mathrm{Si}$ & 150 & - & 一 & 一 & $\mathrm{A}, \mathrm{N}$ \\
\hline Small Herschelian $\eta$ & WC/C & 54.7 & - & - & 一 & $A, N$ \\
\hline Small Herschelian $\theta$ & $\mathrm{Rh} / \mathrm{C}$ & 44.1 & - & - & - & $\mathrm{N}$ \\
\hline $\begin{array}{ll}\text { Comments: } & \text { A - } \\
& \text { C - } \\
& \text { N - }\end{array}$ & $\begin{array}{l}\text { system (no } \\
\text { t SSRL } 199 \\
\text { are present }\end{array}$ & $\begin{array}{l}\text { in 1991) } \\
\text { his paper }\end{array}$ & $\begin{array}{l}\mathrm{B}-\mathrm{Me} \\
\mathrm{D}-\mathrm{Me} \\
\mathrm{Z}-\mathrm{Min}\end{array}$ & ating ha & earch & egments. \\
\hline
\end{tabular}

\section{NEW RESULTS}

During March and April of 1994 we were graciously granted a long section of beam time to calibrate our higher energy $\left(\lambda_{0}<130 \AA\right)$ and newly fabricated instruments. 


\subsection{Experimental Setup}

In our previous measurements at SSRL the detector used was a photocathode. The first set of measurements were made using a gold photocathode which was switched in favor of a polished stainless steel photocathode in later measurements for greater surface uniformity. The photoelectrons produced by this material were collected by an anode at high voltage, and the current was measured using a Keithley 427 current to voltage amplifier.

The low quantum efficiency of the photocathode led to the design of the setup shown in figure 1 . The detector used was a simple photodiode.

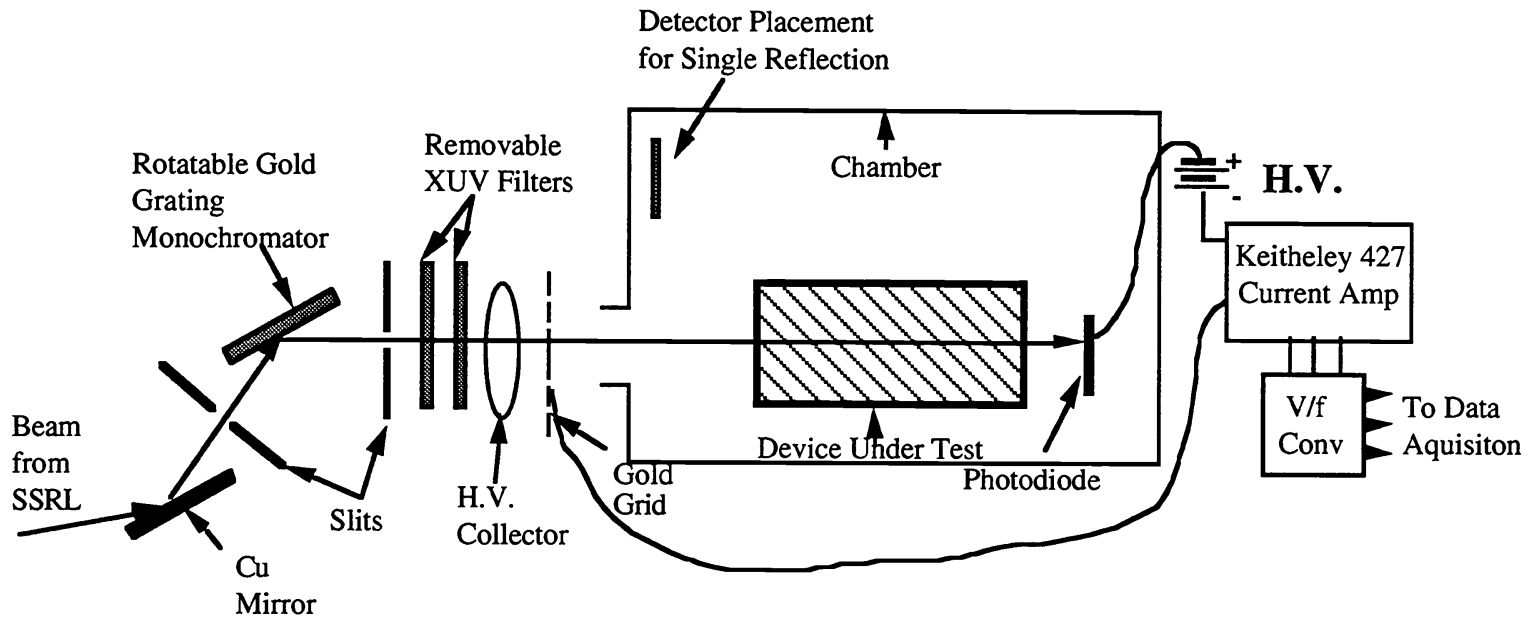

Figure 1. Block diagram of the experimental set-up at SSRL.

An important element of the set-up not shown in the diagram is the SSRL developed differential pumping system which coupled our chamber to the beamline. Due to leakage caused by using an outdated non-UHV chamber, our CTI Cryo-8 pump allowed us to reach only $\sim 10^{-7}$ torr after several hours of pumping. This was not enough to allow us to connect directly to the UHV beamline which was at $10^{-9}$ torr. P. Pianetta and W. Warburton have developed a differential pump which can support up to four orders of magnitude pressure gradient at these pressures ${ }^{7}$. We are indebted to SSRL for making this pumping system available to us.

\subsection{Analysis}

Device throughputs are given simply by the ratio of the photocurrent obtained with the device in the beam to the photocurrent obtained with the device removed. This simple strategy is complicated by several factors.

The first complication is that measurements made with the device in place are not made concurrently with calibration measurements in which the device has been removed from the beam. This introduces a systematic error because the output flux of the synchrotron is time dependent. This problem is corrected by the use of a $\sim 90 \%$ transmissive gold mesh at the entrance to the calibration chamber. The signal from this detector provides a monitor of incident synchrotron flux. Since the time dependence of the synchrotron source is primarily a slow loss of beam current, we assume that the synchrotron flux at all wavelengths varies similarly with time, viz.:

$$
I_{1}^{c}(\lambda, t)=D(\lambda) M_{1}(\lambda) T_{0} S(\lambda, t)=D(\lambda) M_{1}(\lambda) T_{0} S(\lambda) f(t)
$$




$$
\begin{aligned}
& I_{1}^{f}(\lambda, t)=X(\lambda) D(\lambda) M_{1}(\lambda) T_{0} S(\lambda, t)=D(\lambda) M_{1}(\lambda) T_{o} S(\lambda) f(t) \\
& I_{0}^{c}(\lambda, t)=I_{0}^{f}(\lambda, t)=D_{0}(\lambda) M_{1}(\lambda)\left(1-T_{0}\right) S(\lambda, t)=D_{0}(\lambda) M_{1}(\lambda)\left(1-T_{0}\right) S(\lambda) f(t)
\end{aligned}
$$

Where $I_{1}^{c}(\lambda, t)$ is the Photodiode current with the device removed, $I_{1}^{f}(\lambda, t)$ is the Photodiode current with the device in place, $I_{0}^{c}(\lambda, t)$ the Mesh current with the device removed, $I_{0}^{f}(\lambda, t)$ is the Mesh current with the device in place, $D(\lambda)$ the Photodiode response, $D_{0}(\lambda)$ the Mesh response, $M_{1}(\lambda)$ the Monochromator response, $S(\lambda, t)$ the Synchrotron output, $T_{0}$ the Mesh throughput, and $X(\lambda)$.an unknown throughput. If we then look at the ratio $I_{1}(\lambda, t) / I_{0}(\lambda, t)$, we get a cancellation of the time dependence. This ratio is the time independent detector calibration which is used throughout our analysis. In order to obtain $X(\lambda)$ we simply divide the time independent signal with the device in place by the detector calibration,

$$
X(\lambda)=\frac{\text { Signal }}{\text { Calibration }}=\frac{\mathrm{I}_{1}^{\mathrm{f}} / \mathrm{I}_{0}^{\mathrm{f}}}{\mathrm{I}_{1}^{\mathrm{c}} / \mathrm{I}_{0}^{\mathrm{c}}}
$$

The output of the current amplifiers is given by

$$
\mathrm{V}_{i}=\mathrm{G}_{i} \mathrm{I}_{i} \quad i=0,1 \text { corresponding to the two device channels. }
$$

Where $\mathrm{G}_{i}$ are the gains of the amplifiers and $\mathrm{I}_{i}$ are the currents at the Mesh $(i=0)$ and the photodiode $(i=1)$. The outputs of the voltage to frequency converters are given by

$$
\mathrm{f}_{i}=\alpha_{i} \mathrm{~V}_{i}+\beta_{i} \quad i=0,1 \text { corresponding to the two device channels. }
$$

Where $\alpha_{i}$ is a proportionality constant, $\mathrm{V}_{i}$ is the output of the current amplifier, and $\beta_{i}$ is a constant offset determined by measurement (more on this in section 3.3). The device throughput is then:

$$
X(\lambda)=\frac{\left(\frac{{ }^{f_{1}}{ }^{f}-\beta_{1}^{f}}{G_{1}^{f}}\right) /\left(f_{0} \frac{f^{f}-\beta_{0}^{f}}{G_{0}^{f}}\right)}{\left(\frac{{ }^{f}{ }^{c}-\beta_{1}^{c}}{G_{1}^{c}}\right) /\left(f_{0} \frac{c-\beta_{0}^{c}}{G_{0}^{c}}\right)}
$$

\subsection{Errors}

As in previous measurements, higher order light from the monochromator is the most important source of systematic error (see ref. 9). Thin film filters were used to attenuate higher orders. At SSRL beamline 3-1 we had five filters available; their compositions and thickness are: $\mathrm{Al} 1500 \pm 100 \AA$, $\mathrm{Al}$ with $1 \% \mathrm{Si} / \mathrm{Ti}$ overcoat $1500 \pm 100 \AA / 270 \pm 20 \AA$, Al with $1 \%$ Si $1500 \pm 100 \AA$, B $1200 \pm 100 \AA$, and C $1200 \pm 100 \AA$. This provided a broad selection of filters with absorption edges appropriate for almost any chosen wavelength band. For example, the data for fig. $2 \mathrm{~b}$ was taken using an aluminum filter (the aluminum edge is at $171 \AA$ ). It is evident from the peak at $\sim 295 \AA$ that about $6 \%$ of the measured flux from the monochromator was second-order light. If the same percentage of second-order light holds throughout the range of the monochromator, this would translate directly into a $6 \%$ underestimate of peak reflectivity for every mirror. In reality, the second-order contribution is wavelength-dependent, and we have not attempted to apply any correction.

The environment at beamline 3-1 was electrically noisier than that at beamline 1-2 where our previous measurements were carried out. Shortening the detector signal lines helped only a little, and finally it was necessary to lower the gain of the current amplifiers and increase integration times to $3-7$ seconds. The effects of random noise are evident only in our most sensitive measurements (figs. 2a, 5b). 
At low signal levels, random noise can cause the number of counts per second coming from the v-f converter to dip below the offset level, leading to a negative value in either the numerator or the denominator (or both) of equation 7 . Since the lowest signal levels were always from the diode during the data run (this is the factor at top left in eq. 7), the principal manifestation of this problem is in small negative reflectivities measured at the low end of the dynamic range for a few of the curves. Other than this, offset problems are unimportant except for the measurement of the third-order peak of the $150 \AA$ Ritchey-Chrétien primary mirror (fig. 2a), which is not quantitatively very useful since it is very sensitive to the determination of the offset.

It was observed that many of the runs taken with short integration time at coarse resolution are very noisy, and miss some of the major features in the device response. We suspect that either the monochromator or the electronics had some sort of slewing problem. There was no evidence of such difficulty in the more careful, high resolution scans used for our data analysis, but future calibration work should include an attempt to isolate and control this problem.

\subsection{Results and Discussion}

Figures 1 through 6 show reflectivity curves for all of the flight mirrors which were measured. All four of the Mo/Si mirrors had high peak reflectivities of 20\%-35\%. Previous measurements of the $150 \AA$ Ritchey-Chretien telescope (given in table 1) included both mirrors, so I have simulated a composite measurement in fig. $6 \mathrm{~b}$ for comparison. The 1994 measurement shows much lower throughput (10.1\%) than our 1992 measurement. This may be symptomatic of interdiffusion of the molybdenum and silicon layers over the course of time, or it may instead be due to surface contamination. The FWHM of $6.2 \AA$ is virtually identical to the 1992 measurement (see table 1), and the peak wavelength appears to have changed from $152.5 \AA$ to $151.4 \AA$. Of course, the discrepancy in the wavelength measurements is probably due to small differences among the various segments of the primary mirror, which was coated in three passes. In any case, it appears that we have observed a very significant degradation of the mirror reflectivity between 1992 and 1994.

The $54.7 \AA$ and $44 \AA$ mirrors (figs. 5, 6) were less reflective than expected (3.4\% and $2.5 \%$, respectively) but came in very close to the design-specified bandpasses.

All of the Mo/Si multilayers exhibit an asymmetry in specular reflectivity. At wavelengths shorter than the principal bandpass, the reflectivity is negligible-typically $0.1 \%$ or less; but longward of the bandpass, the reflectivity is about $1 \%-1.5 \%$ (see especially fig. $2 \mathrm{~b}$ ). This characteristic is an important insight for designers of XUV optical systems for solar observations. When choosing lines or line multiplets for observation, contamination due to other spectral lines at very different temperatures is always a consideration. Knowing that the multilayer will probably be far more effective at rejecting contaminant lines shortward of the bandpass is very helpful.

The measured second- and third-order reflectivities were extremely low (figs. 1b, 2a, 4a, 5b). This can be attributed to the presence of absorption edges in the multilayer constituents, namely the silicon edge at $123 \AA$ for the $150 \AA \mathrm{Mo} / \mathrm{Si}$ mirrors, and the carbon edge at $43 \AA$ for the $44 \AA \mathrm{Rh} / \mathrm{C}$ mirror. Although not measured, we would expect the same trait in the $54.7 \AA \mathrm{WC} / \mathrm{C}$ mirror. 

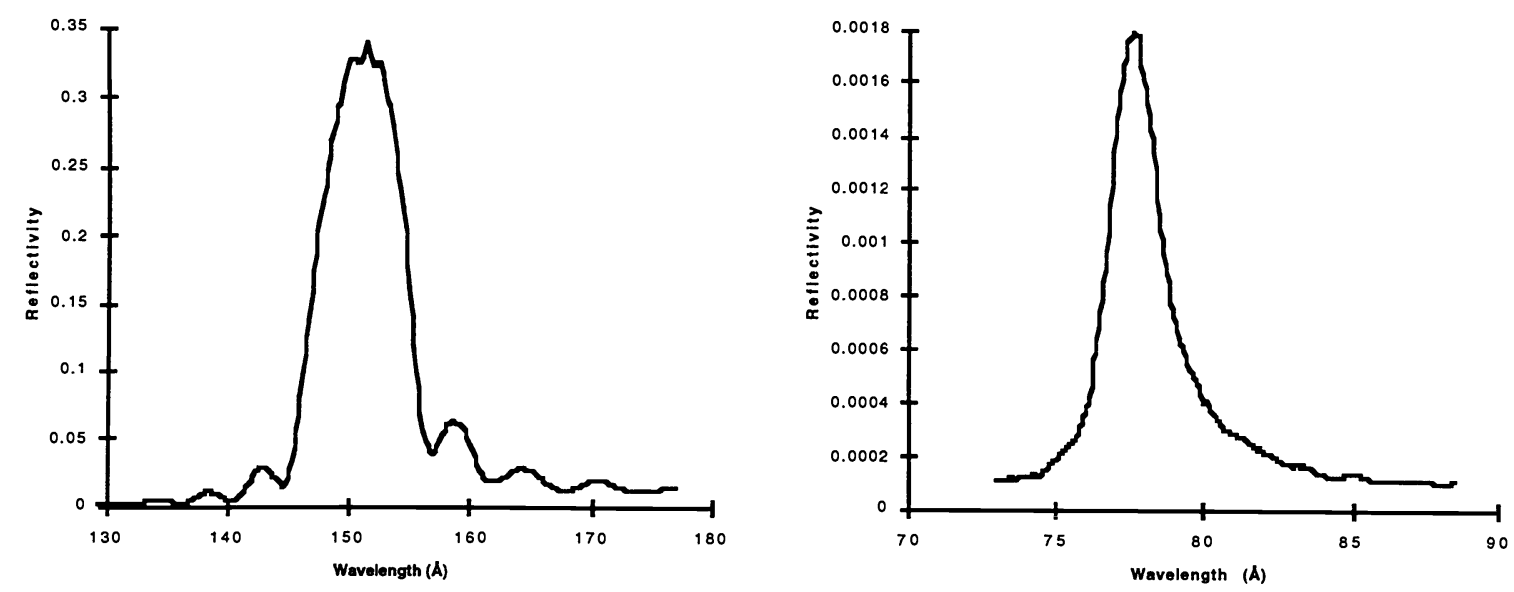

Fig. 1. Reflectivity of the $150 \AA$ (O V/VI) Ritchey-Chrétien primary mirror. At left is the primary bandpass. The curve at right shows reflectivity in second order, which is greatly diminished because the multilayer coating includes silicon, with an absorption edge at $123 \AA$.
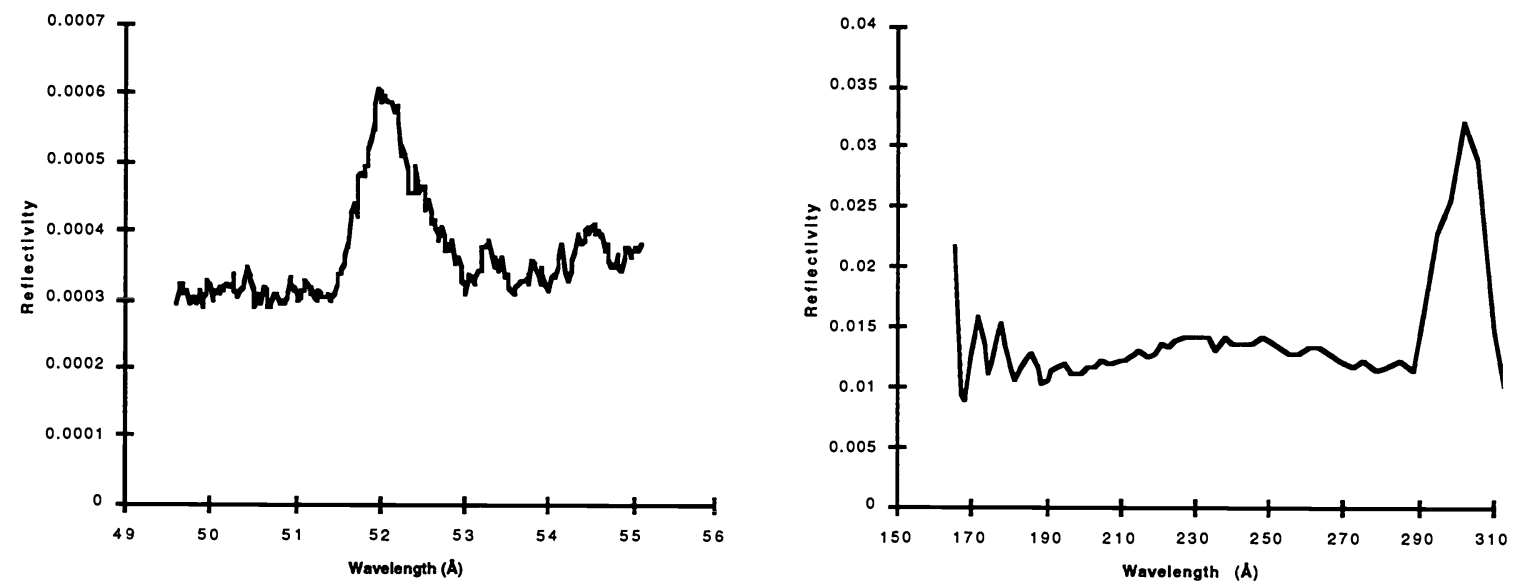

Fig. 2. Reflectivity of the $150 \AA$ Ritchey-Chrétien primary mirror. The curve at left shows third order reflectivity. At right is specular reflectivity longward of the primary bandpass. The peak at $\sim 295 \AA$ is due to $150 \AA$ A light passed in second order by the monochromator. 

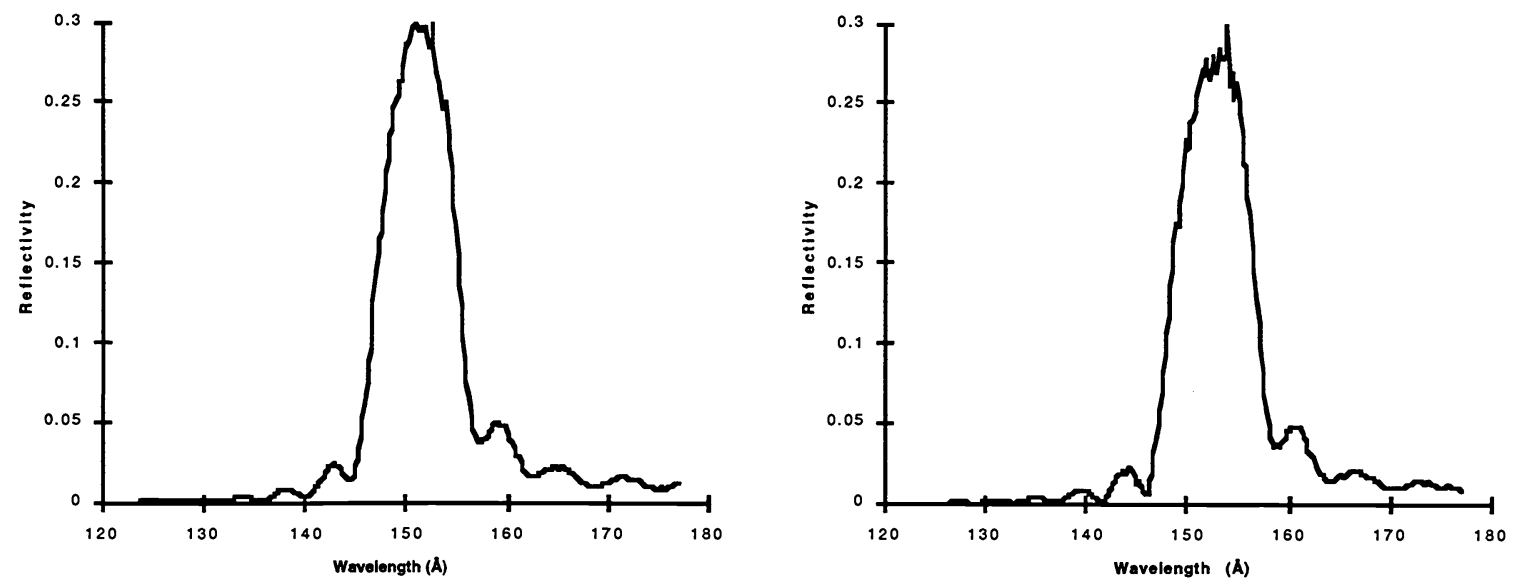

Fig. 3. Reflectivity of the $150 \AA$ A Ritchey-Chrétien secondary mirror. Data for the two reflectivity curves were taken from two widely separated spots on the same mirror. The curves are identical in shape but are offset by $1.5 \AA$.
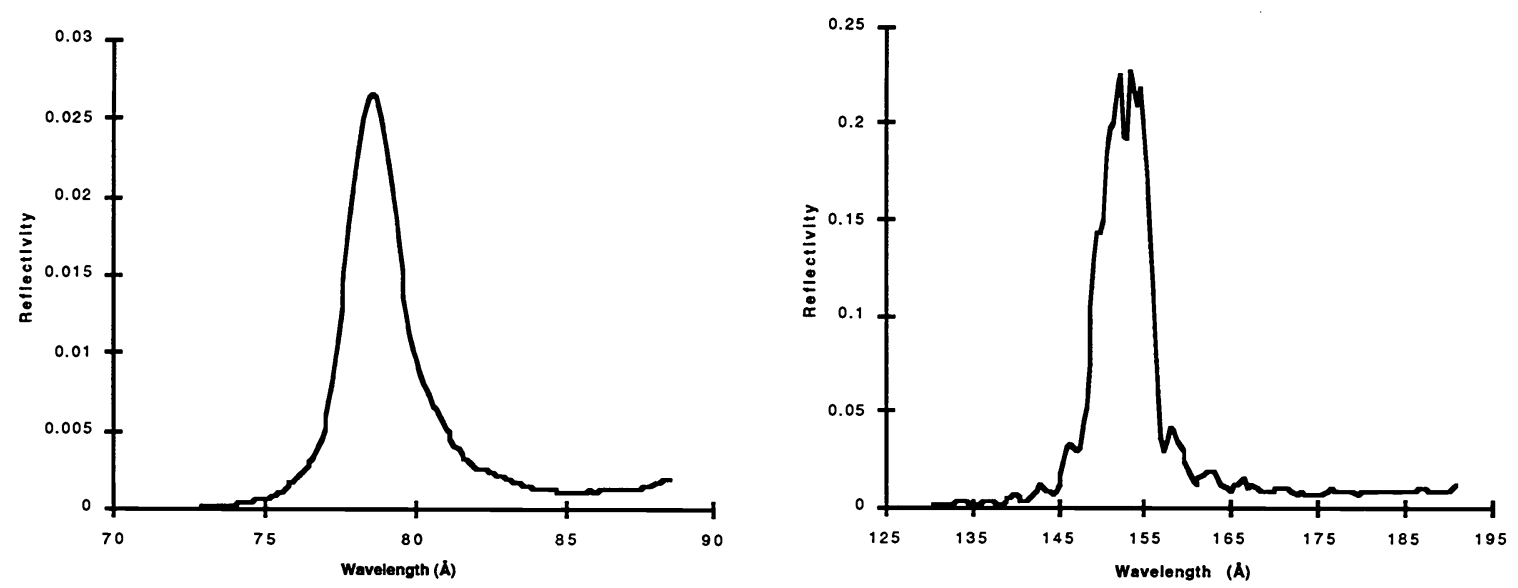

Fig. 4. Left: second-order reflectivity curve for the $150 \AA$ Ritchey-Chrétien secondary mirror. Right: principal bandpass of the $150 \AA$ Aerschelian telescope. 

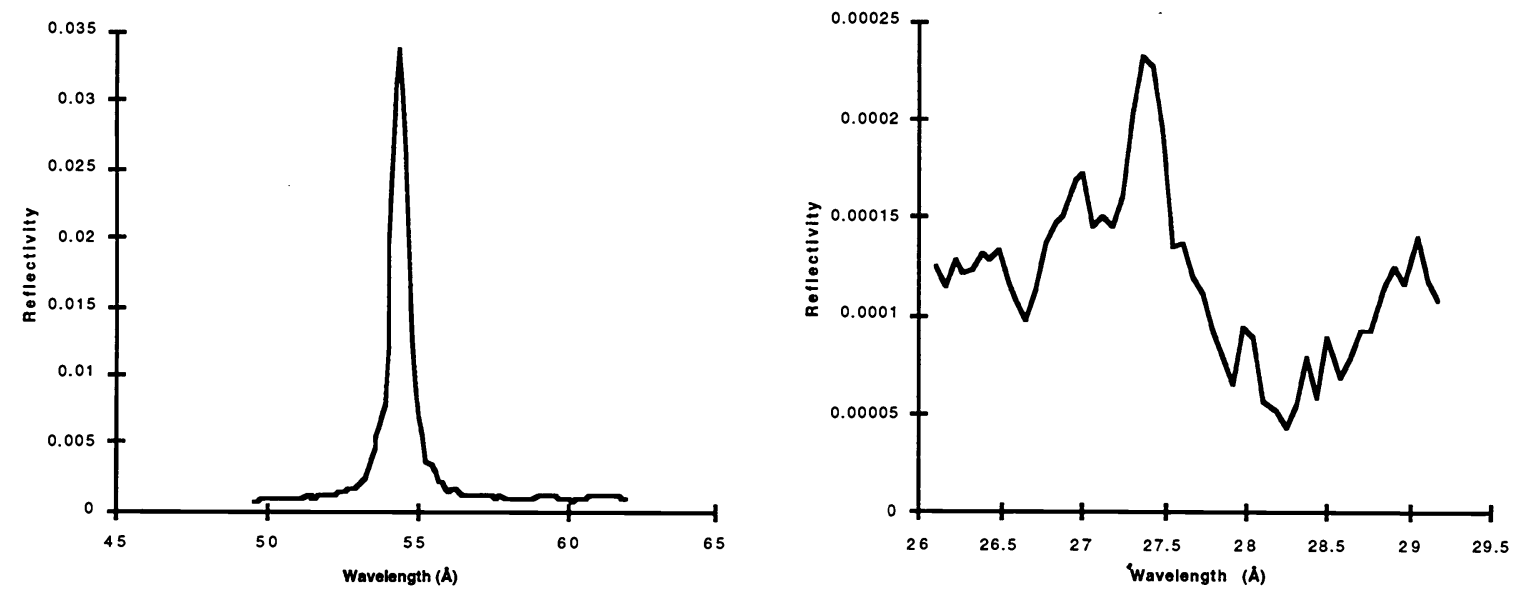

Fig. 5. Reflectivity of the WC/C multilayer $54.7 \AA$ (Fe XVI) Herschelian telescope. Left: principal bandpass. Right: Second order peak, barely detectable in the noise.
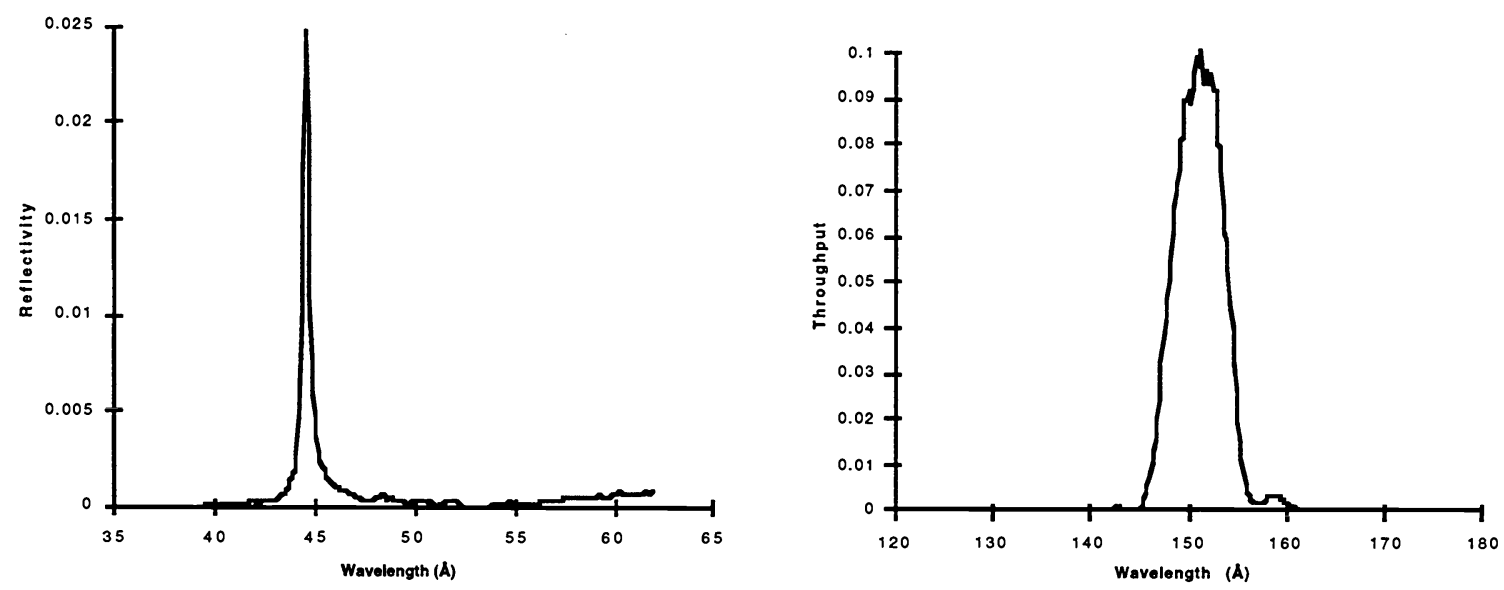

Fig. 6. Left: $44 \AA$ (Si XII) Herschelian mirror. Right: composite reflectivity of the $150 \AA$ Ritchey-Chrétien.

\section{ACKNOWLEDGMENTS}

The MSSTA project at Stanford University is supported by NASA Grant NSG-5131. Richard B. Hoover is supported in part by a grant from the MSFC Center Director's Discretionary Fund and by NASA Grant NSG-5131. Troy W. Barbee, Jr., is supported by the U.S. Department of Energy through Lawrence Livermore National Laboratory under contract W-7405-Eng-48. Work done partially at SSRL which is operated by the Department of Energy, Division of Chemical Sciences. We would like to thank Piero A. Pianetta and Michael Rowen of SSRL for excellent advice and support. We thank everyone at SSRL for making it such a useful and user-friendly facility. 


\section{REFERENCES}

1. A.B.C. Walker, Jr., J.F. Lindblom, R.H. O'Neal, R.B. Hoover, and T.W. Barbee, Jr., "Astronomical observations with normal incidence multilayer optics: Present results and future prospects," Phys Scripta. 41, $1053,1990$.

2. A.B.C. Walker, Jr., R.B. Hoover and T.W. Barbee, Jr., "High Resolution Thermally Differentiated Images of the Chromosphere and Corona," to be publ. in Advances in Stellar and Solar Coronal Physics: Proc. of the Vacana Memorial Symposium, J. Linsky, Ed. Kluwer Acad. Publ. Dordrecht 1993; A.B.C. Walker, Jr., T.W. Barbee, Jr., and R.B. Hoover, "Astronomical Observations with Normal Incidence Multilayer Optics II: Images of the Solar Corona and Chromosphere," UV and X-Ray Spectroscopy of Laboratory and Astrophysical Plasmas: Proc. of the Tenth IAU International Colloquium on Laboratory and Astrophysical Plasmas, Berkeley, CA 1992, E. Silver and S. Kahn, Eds., Cambridge Univ. Press, 1993, p. 193.

3. A. B.C. Walker, Jr., L. Jackson , J. Plummer, Richard B. Hoover, and Troy W. Barbee Jr. "Astronomical Observations with Normal Incidence Mutilayer Optics III: Selection of Multilayer Bandpasses," Proc. SPIE 2011, 450, 1993.

4. J.E.Plummer, C.E. DeForest, D. Martínez-Galarce, C.C. Kankelborg, D. Gore, R. O'Neal, A.B.C. Walker, F. Powell, R.B. Hoover, T.W. Barbee, "The design and performance of thin foil XUV filters for the Multi-Spectral Solar Telescope Array II," Proc. SPIE 2515, 1995.

5. R.B. Hoover, A.B.C. Walker, jr., C.E. DeForest, M.J. Allen, J.F. Lindblom, "EUV/FUV response characteristics of photographic films for the Multi-Spectral Solar Telescope Array," Proc. SPIE 2011, 1993.

6. C.E. DeForest, C.C. Kankelborg, M.J. Allen, E.S. Paris, T.D. Willis, J.F. Lindblom, R.H. O'Neal, A.B.C. Walker, Jr., T.W. Barbee, Jr. and R.B. Hoover, "The Multi-Spectral Solar Telescope Array V: Temperature diagnostic response to the optically thin solar plasma," Optical Eng. 30, 1125, 1991.

7. W.K. Warburton, and P. Pianetta, "A Novel Differential Pump for Synchrotron Beamlines: Tests, Models and Applications.," Nucl. Instr. and Meth. A291, p 350-356, 1990.

8. M. J. Allen, T. D. Willis et al, "Calibration of the Multi-Spectral Solar Telescope Array Multilayer Mirrors and Filters," Proc. SPIE 1742, 562, 1992.

9. M. Allen, T.D. Willis, C.C. Kankelborg, R.H. O'Neal, D.S. Martínez-Galarce, C.E. Deforest, L.R. Jackson, J.Plummer, A.B.C. Walker, Jr., T. Barbee, Jr., J.W. Weed, and R. Hoover, "Performance of the Multilayer Coated Mirrors for the Multi-Spectral Solar Telescope Array," Proc. SPIE 2011, 1993. 